\title{
Enrichment post-library preparation enhances the sensitivity of high- throughput sequencing-based detection and characterization of viruses from complex samples
}

\author{
Adrian C. Paskey ${ }^{1,2,3}$, Kenneth G. Frey ${ }^{1}$, Gary Schroth ${ }^{4}$, Stephen Gross ${ }^{4}$, Theron Hamilton ${ }^{1}$ and \\ Kimberly A. Bishop-Lilly ${ }^{1 *}$
}

\begin{abstract}
Background: Sequencing-based detection and characterization of viruses in complex samples can suffer from lack of sensitivity due to a variety of factors including, but not limited to, low titer, small genome size, and contribution of host or environmental nucleic acids. Hybridization-based target enrichment is one potential method for increasing the sensitivity of viral detection via high-throughput sequencing.

Results: This study expands upon two previously developed panels of virus enrichment probes (for filoviruses and for respiratory viruses) to include other viruses of biodefense and/or biosurveillance concern to the U.S. Department of Defense and various international public health agencies. The newly expanded and combined panel is tested using carefully constructed synthetic metagenomic samples that contain clinically relevant amounts of viral genetic material. Target enrichment results in a dramatic increase in sensitivity for virus detection as compared to shotgun sequencing, yielding full, deeply covered viral genomes from materials with Ct values suggesting that amplicon sequencing would be likely to fail. Increased pooling to improve cost- and time-effectiveness does not negatively affect the ability to obtain full-length viral genomes, even in the case of co-infections, although as expected, it does decrease depth of coverage.

Conclusions: Hybridization-based target enrichment is an effective solution to obtain full-length viral genomes for samples from which virus detection would fail via unbiased, shotgun sequencing or even via amplicon sequencing. As the development and testing of probe sets for viral target enrichment expands and continues, the application of this technique, in conjunction with deeper pooling strategies, could make high-throughput sequencing more economical for routine use in biosurveillance, biodefense and outbreak investigations.
\end{abstract}

Keywords: Genome enrichment, Virus detection, High-throughput sequencing, Virome profiling, Virome capture sequencing, Viral enrichment, Sequencing-based detection, Next generation sequencing, Hybridization-based target enrichment

\footnotetext{
* Correspondence: Kimberly.a.bishop-lilly.civ@mail.mil

${ }^{1}$ Genomics and Bioinformatics Department, Biological Defense Research

Directorate, Naval Medical Research Center - Frederick, Fort Detrick,

Frederick, MD 21702, USA

Full list of author information is available at the end of the article
}

(c) The Author(s). 2019 Open Access This article is distributed under the terms of the Creative Commons Attribution 4.0 International License (http://creativecommons.org/licenses/by/4.0/), which permits unrestricted use, distribution, and reproduction in any medium, provided you give appropriate credit to the original author(s) and the source, provide a link to the Creative Commons license, and indicate if changes were made. The Creative Commons Public Domain Dedication waiver (http://creativecommons.org/publicdomain/zero/1.0/) applies to the data made available in this article, unless otherwise stated. 


\section{Background}

High-Throughput Sequencing (HTS), also known as Next-Generation Sequencing (NGS), has many advantages for pathogen detection as compared to traditional methods such as Polymerase Chain Reaction (PCR), serological assays, and/or culture-based methods. Metagenomic sequencing is the high-throughput sequencing of nucleic acid from complex samples rather than from purified microorganisms. Metagenomic sequencing is much less biased than other methods and allows for the detection of fastidious or nonculturable organisms as well as multiple unrelated pathogens within a single sample [1]. Moreover, detection via HTS is much less susceptible to false-negative results caused by antigenic drift or signature erosion. Despite these advantages, one of the technical challenges encountered with respect to metagenomic sequencing is obtaining adequate depth and breadth of coverage from pathogens like RNA viruses that i) typically have small genomes, and ii) are typically present at low titers amidst the background 'noise' of the host and commensals [1]. Genome size directly affects sensitivity of detection by HTS because the sampling of sequence fragments within a sample depends on the prevalence of those fragments and organisms with larger genomes typically contribute more fragments, therefore being sampled more often than organisms with smaller genomes. In other words, organisms with larger genomes have the potential to contribute a larger proportion to the overall number of sequencing reads even when the plaque-forming units (PFU), or colony-forming units (CFU) in the case of bacteria, are equivalent to that of an organism with a smaller sized genome.

Although conventional shotgun sequencing allows for the detection of all domains of life, it rarely returns robust coverage of a small viral genome when taken from a very complex sample. A variety of possible strategies exist to enhance the sensitivity of HTS for virus detection and characterization, including purification of specific viral fractions by physical methods such as filtration and ultracentrifugation [2], amplicon-based target enrichment, and hybridization-based target enrichment. Purification of viral fractions is ideal in some cases, although it can be laborious, and for certain sized samples (for instance clinical samples of very limited volume) it may not be realistic. The use of hybridization-based target enrichment could be preferable to the aforementioned technologies because it has the potential to yield sequence data covering the entire genome of multiple viruses with just one sequencing reaction by using genome-wide probes designed against multiple viruses to specifically select for viral cDNA prior to sequencing. Amplicon sequencing of viral genomes is a technique that has been widely used, but it has some disadvantages, which were articulated by Metsky et al. in a recent study of Zika virus (ZIKV) [3]. First, traditional amplicon sequencing typically requires technically challenging normalization and pooling of individual amplicons to cover the entire genome of one specific virus. However, recently a protocol was published for efficient amplicon primer design and multiplex amplicon generation in a single tube for sequencing in the MinION or Illumina platforms [4]. Although this method obviates the amplicon normalization and pooling steps and is effective for producing whole genome sequence data from a low titer ZIKV sample, this method has not been demonstrated for production of whole genome sequence data for multiple diverse viruses from a single complex sample. Additionally, amplicon sequencing typically requires as much as $40 \mathrm{cy}$ cles of PCR amplification [4-6], which can introduce sequence errors. Furthermore, amplicon-based sequencing is vulnerable to false negative results caused by mutations in primer binding sites, as was recently demonstrated for Dengue virus (DENV) [5] as well as false positive variant results possibly caused by low and/or uneven coverage [7]. By contrast, the use of probes tiled along the entire length of a viral genome to hybridize and select for virus-specific fragments has the potential to produce less false-negative pathogen detection results by virtue of many more potential binding sites along an individual genome and resulting more uniform coverage.

Ebola virus (EBOV) is one specific example of a pathogen for which false-negative PCR results can have devastating consequences and for which available PCR-based assay effectiveness has been shown to be affected by drift [8]. Therefore, a panel of 80-mer oligonucleotide probes designed against eight Filovirus genomes was recently used for post-sequencing library enrichment in a HTSbased study of a recent EBOV outbreak in West Africa and in an investigation of potential genetic variation of EBOV in experimentally infected nonhuman primates [9-11]. In this protocol, viral enrichment is coupled with the RNA Access kit, developed by Illumina, Inc. The technical advancements of the RNA Access kit had already enabled the sequencing of previously unsequencable materials such as those of low concentration and formalin-fixed paraffin-embedded (FFPE) tissue [12], and now this protocol has been employed not only for the detection and characterization of EBOV from clinical samples but also for detection and characterization of respiratory viruses in clinical samples $[13,14]$. In general, hybridization-based viral target enrichment has been successfully employed to characterize viruses found within both contrived samples and clinical samples [3, 9, 10, 13, 15-20]. The performance of the Respiratory Virus Panel (RVP) version of this method [14], which uses probes for 34 common respiratory viruses in conjunction with the TruSeq RNA Access protocol, was 
recently investigated and it was demonstrated to work well overall when tested on human clinical samples [13]. Specifically, the authors reported successful enrichment for 30 of 33 human clinical samples tested. Importantly, RT-PCR was conducted on those same samples and $\mathrm{Ct}$ values of respiratory viruses in those clinical samples ranged from 21 to 33 [13], which provides a framework for beginning to assess the limits of detection of hybridization-based enrichment sequencing. Herein, we extend this approach by i) expanding this viral probe panel to include viruses of biosurveillance and biodefense concern and ii) employing carefully constructed mock clinical samples to systematically assess this technique's performance in a variety of conditions, such as deeper multiplexing for cost effectiveness as well as more extensive co-infection scenarios. We demonstrate the sensitivity and reproducibility of hybridization-based viral enrichment sequencing despite virus divergence and we show that this sensitivity is maintained even with extensive multiplexing of samples to decrease cost. Herein we demonstrate that within one reaction tube, this technique can even be used to detect and discriminate between multiple serotypes of a virus within a clinical sample or to detect and discriminate amongst multiple unrelated viruses that present similar clinical symptoms, and we demonstrate this performance at clinically relevant concentrations of virus.

\section{Results}

Hybridization-based target enrichment enhances sensitivity of HTS for detection of virus in complex environmental samples

Enhanced detection of viruses from various clinical sample types using filovirus- or respiratory virus-specific probes has recently been demonstrated $[9,10,13]$. To expand the range of viruses that could be detected, in this study those two probe panels were combined with new probes for 41 additional viruses that are of biosurveillance and biodefense concern, for a full panel targeting 83 diverse viruses (Table 1). In order to test this newly expanded probe panel and to specifically assess the effect of hybridization-based viral enrichment on the sensitivity of HTS for detection of a single virus within a complex environmental sample, commercial bat guano was spiked with increasing concentrations of Influenza virus (IFV). Spiked samples were split into two parts each, with each part being processed in parallel with unbiased, shotgun sequencing versus target enrichment sequencing using an expanded panel of probes.

As expected, a dose-dependent effect in the proportion of sequencing reads derived from IFV was observed as the number of spiked genome copies increased (Fig. 1a and Additional file 1: Table S1), in both the unbiased shotgun sequence data as well as the virus enriched sequence data. However, in this context, hybridizationbased target enrichment resulted in approximately 20- to 100-fold more sensitivity for detection of IFV as compared to detection via unbiased, shotgun sequencing. At the lowest concentration tested (1250 genome equivalents (GE) per $\mathrm{mL}$ ), only $0.5 \%$ of sequencing reads produced by unbiased shotgun sequencing were derived from IFV ('on target' reads), whereas by stark contrast, the majority of reads produced by target enrichment sequencing (54.4\%) were derived from IFV.

Given the dramatic increase in sensitivity observed when complex samples were spiked with an individual virus's genetic material and subjected to target enrichment, we next sought to evaluate whether these effects would still be observed in the presence of an additional virus and at lower concentrations of IFV gRNA overall. Therefore, IFV gRNA was spiked into total RNA derived from Middle Eastern Respiratory Syndrome Coronavirus (MERS-CoV) cell culture lysate at an overall lower range of increasing concentrations than IFV was spiked in the prior experiment. As before, the samples were aliquoted into two parts that were processed by each method. In these synthetic co-infection samples, target enrichment sequencing resulted in a simultaneous increase in sensitivity for both viruses as compared to unbiased, shotgun sequencing (Fig. 1b). As expected, a constant high proportion of reads mapping to MERS-CoV was observed and the proportion of reads mapping to IFV increased in a dose-dependent fashion with the number of genome equivalents spiked (Additional file 2: Table S2).

\section{Detection and discrimination of related viruses in clinical samples}

We next tested the sensitivity for detection of three clinically-relevant viruses that can co-circulate in tropical regions, can present with similar symptoms, and can be difficult to detect at low titers [21]. Mock clinical samples were constructed containing combinations of ZIKV, CHIKV, and DENV at loads that correlate with real clinical loads from human specimens. Briefly, varying titers of ZIKV, Dengue virus 2 (DENV-2), and Chikungunya virus (CHIKV) were spiked into RNA extracted from human serum, in duplicate, to create synthetic co-infection samples. Negative control samples consisted solely of RNA extracted from human serum. Viruses were spiked-in at concentrations corresponding to $\mathrm{Ct}$ values from standard curves generated via RTqPCR. The targeted spike-in values were chosen based on reports in the literature for clinical samples containing each virus to mimic a realistic co-infection scenario [22-25]. Given that in the literature there is at least one report of clinical samples being probed singly rather than pooled and probed [9] and it is not well known how pooling may affect virus detection levels, in this 
Table 1 Viruses included in target enrichment panel

\begin{tabular}{|c|c|c|c|c|}
\hline Virus & Genome size (kb) & Genome type & $\begin{array}{l}\text { NCBI accession(s) of reference } \\
\text { used in probe design }\end{array}$ & Notes \\
\hline Nipah virus & 18,246 & Negative sense ssRNA & NC_002728.1 & New addition to probe panel \\
\hline Bat Paramyxovirus & 18,530 & Negative sense ssRNA & NC_025256.1 & New addition to probe panel \\
\hline Cedar virus & 18,162 & Negative sense ssRNA & JQ001776.1 & New addition to probe panel \\
\hline Hendra virus & 18,234 & Negative sense ssRNA & NC_001906.3 & New addition to probe panel \\
\hline Tioman virus & 15,522 & Negative sense ssRNA & NC_004074.1 & New addition to probe panel \\
\hline Menangle virus & 15,516 & Negative sense ssRNA & NC_007620.1 & New addition to probe panel \\
\hline $\begin{array}{l}\text { Middle East Respiratory } \\
\text { Syndrome Coronavirus }\end{array}$ & 30,094 & Positive sense ssRNA & KJ614529.1 & New addition to probe panel \\
\hline $\begin{array}{l}\text { Severe Acute Respiratory } \\
\text { Syndrome virus }\end{array}$ & 29,751 & Positive sense ssRNA & NC_004718.3 & New addition to probe panel \\
\hline Lujo virus & 10,352 & Negative sense ssRNA & NC_012776.1, NC_012777.1 & New addition to probe panel \\
\hline Lassa fever virus & 10,681 & Negative sense ssRNA & NC_004296.1, NC_004297.1 & New addition to probe panel \\
\hline Machupo virus & 10,635 & Negative sense ssRNA & NC_005078.1, NC_005079.1 & New addition to probe panel \\
\hline Junin virus & 10,525 & Negative sense ssRNA & NC_005080.1, NC_005081.1 & New addition to probe panel \\
\hline Guanarito virus & 10,424 & Negative sense ssRNA & NC_005077.1, NC_005082.1 & New addition to probe panel \\
\hline Chapare virus & 10,464 & Negative sense ssRNA & NC_010562.1, NC_010563.1 & New addition to probe panel \\
\hline Sabia virus & 10,499 & Negative sense ssRNA & NC_006313.1, NC_006317.1 & New addition to probe panel \\
\hline Hantaan virus & 11,845 & Negative sense ssRNA & $\begin{array}{l}\text { NC_005218.1, NC_005219.1, } \\
\text { NC_005222.1 }\end{array}$ & New addition to probe panel \\
\hline Puumala virus & 12,062 & Negative sense ssRNA & $\begin{array}{l}\text { NC_005223.1, NC_005224.1, } \\
\text { NC_005225.1 }\end{array}$ & New addition to probe panel \\
\hline Sin nombre virus & 12,317 & Negative sense ssRNA & $\begin{array}{l}\text { NC_005215.1, NC_005216.1, } \\
\text { NC_005217.1 }\end{array}$ & New addition to probe panel \\
\hline Andes virus & 12,104 & Negative sense ssRNA & $\begin{array}{l}\text { NC_003466.1, NC_003467.1, } \\
\text { NC_003468.1 }\end{array}$ & New addition to probe panel \\
\hline Rift Valley fever virus & 11,979 & Negative sense ssRNA & $\begin{array}{l}\text { NC_014395.1, NC_014396.1, } \\
\text { NC_014397.1 }\end{array}$ & New addition to probe panel \\
\hline $\begin{array}{l}\text { Crimean Congo hemorrhagic } \\
\text { fever virus }\end{array}$ & 19,146 & Negative sense ssRNA & $\begin{array}{l}\text { NC_005300.2, NC_005301.3, } \\
\text { NC_005302.1 }\end{array}$ & New addition to probe panel \\
\hline Omsk hemorrhagic fever virus & 10,787 & Positive sense ssRNA & NC_005062.1 & New addition to probe panel \\
\hline Kyasanur forest disease virus & 10,774 & Positive sense ssRNA & $J F 416958.1$ & New addition to probe panel \\
\hline $\begin{array}{l}\text { Alkhurma hemorrhagic } \\
\text { fever virus }\end{array}$ & 10,685 & Positive sense ssRNA & NC_004355.1 & New addition to probe panel \\
\hline Eastern equine encephalitis virus & 11,703 & Positive sense ssRNA & KJ469643.1 & New addition to probe panel \\
\hline Dengue type 1 virus & 10,721 & Positive sense ssRNA & AF309641.1 & New addition to probe panel \\
\hline Dengue type 2 virus & 10,723 & Positive sense ssRNA & EF051521.1 & New addition to probe panel \\
\hline Dengue type 3 virus & 10,707 & Positive sense ssRNA & AY662691 & New addition to probe panel \\
\hline Dengue type 4 virus & 10,653 & Positive sense ssRNA & AY618989 & New addition to probe panel \\
\hline Chikungunya virus & 11,826 & Positive sense ssRNA & NC_004162 & New addition to probe panel \\
\hline Bat coronavirus CDPHE15 & 28,035 & Positive sense ssRNA & NC_022103.1 & New addition to probe panel \\
\hline Bat coronavirus $1 \mathrm{~A}$ & 28,326 & Positive sense ssRNA & NC_010437.1 & New addition to probe panel \\
\hline Bat coronavirus 1B & 28,476 & Positive sense ssRNA & NC_010436.1 & New addition to probe panel \\
\hline Bat coronavirus HKU2 & 27,165 & Positive sense ssRNA & NC_009988.1 & New addition to probe panel \\
\hline Bat SARS coronavirus HKU3-4 & 29,704 & Positive sense ssRNA & GQ153539.1 & New addition to probe panel \\
\hline Bat coronavirus HKU4 & 30,286 & Positive sense ssRNA & NC_009019 & New addition to probe panel \\
\hline Bat coronavirus HKU5-1 & 30,482 & Positive sense ssRNA & NC_009020 & New addition to probe panel \\
\hline Bat coronavirus HKU8 & 28,773 & Positive sense ssRNA & NC_010438.1 & New addition to probe panel \\
\hline
\end{tabular}


Table 1 Viruses included in target enrichment panel (Continued)

\begin{tabular}{|c|c|c|c|c|}
\hline Virus & Genome size $(k b)$ & Genome type & $\begin{array}{l}\text { NCBI accession(s) of reference } \\
\text { used in probe design }\end{array}$ & Notes \\
\hline Bat coronavirus HKU9-1 & 29,114 & Positive sense ssRNA & NC_009021.1 & New addition to probe panel \\
\hline Bat coronavirus HKU10 & 28,494 & Positive sense ssRNA & NC_018871.1 & New addition to probe panel \\
\hline Zika virus & 10,794 & Positive sense ssRNA & NC_012532 & New addition to probe panel \\
\hline Respiratory Syncytial virus B (S2) & 15,190 & Negative sense ssRNA & NC_001803.1 & Previously used in $[13,20]$ \\
\hline Respiratory Syncytial virus A & 15,225 & Negative sense ssRNA & AY353550 & Previously used in $[13,20]$ \\
\hline Influenza virus A (H9N2) & 13,500 & Negative sense ssRNA & $\begin{array}{l}\text { NC_004905.2, NC_004906.1, } \\
\text { NC_004907.1, NC_004908.1, } \\
\text { NC_004909.1, NC_004910.1, } \\
\text { NC_004911.1, NC_004912.1 }\end{array}$ & Previously used in $[13,20]$ \\
\hline Influenza virus A (H2N2) & 13,460 & Negative sense ssRNA & $\begin{array}{l}\text { NC_007374.1, NC_007375.1, } \\
\text { NC_007376.1, NC_007377.1, } \\
\text { NC_007378.1, NC_007380.1, } \\
\text { NC_007381.1, NC_007382.1 }\end{array}$ & Previously used in $[13,20]$ \\
\hline Influenza virus A (H3N2) & 13,630 & Negative sense ssRNA & $\begin{array}{l}\text { NC_007366.1, NC_007367.1, } \\
\text { NC_007368.1, NC_007369.1, } \\
\text { NC_007370.1, NC_007371.1, } \\
\text { NC_007372.1, NC_007373.1 }\end{array}$ & Previously used in $[13,20]$ \\
\hline Influenza virus A (H1N1) & 13,590 & Negative sense ssRNA & $\begin{array}{l}\text { NC_002016.1, NC_002017.1, } \\
\text { NC_002018.1, NC_002019.1, } \\
\text { NC_002020.1, NC_002021.1, } \\
\text { NC_002022.1, NC_002023.1, }\end{array}$ & Previously used in $[13,20]$ \\
\hline Influenza virus A (H5N1) & 13,590 & Negative sense ssRNA & $\begin{array}{l}\text { NC_007357.1, NC_007358.1, } \\
\text { NC_007359.1, NC_007360.1, } \\
\text { NC_007361.1, NC_007362.1, } \\
\text { NC_007363.1, NC_007364.1 }\end{array}$ & Previously used in $[13,20]$ \\
\hline Influenza virus A (H7N9) & 13,590 & Negative sense ssRNA & $\begin{array}{l}\text { KC885955, KC885956, } \\
\text { KC885957, KC885958, } \\
\text { KC885959, KC885960, } \\
\text { KC885961, KC885962 }\end{array}$ & Previously used in $[13,20]$ \\
\hline Influenza virus B & 14,450 & Negative sense ssRNA & $\begin{array}{l}\text { NC_002204.1, NC_002205.1, } \\
\text { NC_002206.1, NC_002207.1, } \\
\text { NC_002208.1, NC_002209.1, } \\
\text { NC_002210.1, NC_002211.1 }\end{array}$ & Previously used in $[13,20]$ \\
\hline Parainfluenza virus 1 & 15,600 & Negative sense ssRNA & NC_003461.1 & Previously used in $[13,20]$ \\
\hline Parainfluenza virus 2 & 15,650 & Negative sense ssRNA & NC_003443.1 & Previously used in $[13,20]$ \\
\hline Parainfluenza virus 3 & 15,460 & Negative sense ssRNA & NC_001796.2 & Previously used in $[13,20]$ \\
\hline Parainfluenza virus 4 & 17,050 & Negative sense ssRNA & NC_021928.1 & Previously used in $[13,20]$ \\
\hline Human metapneumovirus & 13,340 & Negative sense ssRNA & NC_004148.2 & Previously used in $[13,20]$ \\
\hline Adenovirus C & 35,937 & dsDNA & NC_001405.1 & Previously used in $[13,20]$ \\
\hline Adenovirus B & 35,343 & dsDNA & NC_011203.1 & Previously used in $[13,20]$ \\
\hline Adenovirus $\mathrm{E}$ & 35,994 & dsDNA & NC_003266.2 & Previously used in $[13,20]$ \\
\hline Human Coronavirus HKU1 & 29,930 & Positive sense ssRNA & NC_006577.2 & Previously used in $[13,20]$ \\
\hline Human Coronavirus NL63 & 27,550 & Positive sense ssRNA & NC_005831.2 & Previously used in $[13,20]$ \\
\hline Human Coronavirus 229E & 27,320 & Positive sense ssRNA & NC_002645.1 & Previously used in $[13,20]$ \\
\hline Human Coronavirus OC43 & 30,738 & Positive sense ssRNA & AY391777.1 & Previously used in $[13,20]$ \\
\hline Rhinovirus A & 7150 & Positive sense ssRNA & NC_001617.1 & Previously used in $[13,20]$ \\
\hline Rhinovirus C & 7100 & Positive sense ssRNA & NC_001490.1 & Previously used in $[13,20]$ \\
\hline Rhinovirus B14 & 7210 & Positive sense ssRNA & NC_001490.1 & Previously used in $[13,20]$ \\
\hline Human Bocavirus 1 & 5299 & ssDNA & NC_007455.1 & Previously used in $[13,20]$ \\
\hline Human Bocavirus 2 & 5196 & ssDNA & NC_012042.1 & Previously used in $[13,20]$ \\
\hline Human Bocavirus 3 & 5242 & ssDNA & NC_012564.1 & Previously used in $[13,20]$ \\
\hline
\end{tabular}


Table 1 Viruses included in target enrichment panel (Continued)

\begin{tabular}{lllll}
\hline Virus & Genome size (kb) & Genome type & $\begin{array}{l}\text { NCBI accession(s) of reference } \\
\text { used in probe design }\end{array}$ & Notes \\
\hline Human Bocavirus 4 & 5104 & ssDNA & NC_012729.2 & Previously used in [13, 20] \\
KI polyomavirus & 5040 & dsDNA & NC_009238.1 & Previously used in [13, 20] \\
WU polyomavirus & 5229 & dsDNA & NC_009539.1 & Previously used in [13, 20] \\
Human parechovirus type 1 & 7296 & Positive sense ssRNA & FM242866.1 & Previously used in [13, 20] \\
Human parechovirus type 6 & 7347 & Positive sense ssRNA & AB252582.1 & Previously used in [13, 20] \\
Human Enterovirus C104 & 7408 & Positive sense ssRNA & AB686524.1 & Previously used in [13, 20] \\
Human Enterovirus C109 & 7354 & Positive sense ssRNA & GQ865517.1 & Previously used in [13, 20] \\
Lloviu cuevavirus & 18,927 & Negative sense ssRNA & NC_016144 & Previously used in [9-11] \\
Bundibugyo ebolavirus & 18,940 & Negative sense ssRNA & NC_014373 & Previously used in [9-11] \\
Zaire ebolavirus & 18,959 & Negative sense ssRNA & NC_002549 & Previously used in [9-11] \\
Reston ebolavirus & 18,891 & Negative sense ssRNA & NC_004161 & Previously used in [9-11] \\
Sudan ebolavirus & 18,875 & Negative sense ssRNA & NC_006432 & Previously used in [9-11] \\
Tai Forest ebolavirus & 18,935 & Negative sense ssRNA & NC_014372 & Previously used in [9-11] \\
Marburg virus (isolate Marburg virus) & 19,111 & Negative sense ssRNA & NC_001608 & Previously used in [9-11] \\
Marburg virus (isolate Ravn virus) & 19,114 & Negative sense ssRNA & NC_024781 & Previously used in [9-11] \\
\hline
\end{tabular}

experiment we also sought to evaluate whether probing singly or within a pool would affect our ability to identify virus. Therefore, total RNA extracted from these samples was probed singly ("pool of 1") and also pooled in groups of four and 12 with singly-spiked and mock-spiked serum samples consisting of the other components of the pool. The resulting sequence reads were mapped to the reference genomes for each of these three viruses. In all cases, the three co-infecting viruses were able to be detected in each sample at relatively consistent proportions regardless of the number of samples within a pool (Fig. 2a). Even DENV-2 was detectable within each sample it was spiked, despite the low concentration of viral RNA (estimated 100 genome equivalents per $\mathrm{mL}$ ).

Although each targeted virus was represented by enough sequencing reads to be easily detected, there were differences in the depth and breadth of genome coverage observed. Whereas the full CHIKV genome was recovered from all spiked samples at a very high depth of coverage (Fig. 2c), in the case of DENV-2, an average of $92.2 \%$ of the genome was recovered from co-infected samples (Fig. 2d) and the average ZIKV linear genome coverage was lower, at $68.8 \%$ (Fig. 2b). These patterns in coverage were similar for both replicates (Additional file 3: Figure S1). Overall, the proportion of reads mapping to a given virus was consistent and reproducible regardless of whether a sample was probed singly or probed within a group of four or 12 .

In addition to evaluating whether sensitivity and reproducibility are maintained despite multiplexing in pools of four and 12, we also sought to evaluate the probe panel's performance in the context of strain-level, and even species-level, genetic variation as well as differing concentrations of viral genetic material. Specifically, synthetic clinical samples were also constructed to contain a different strain of ZIKV than the strain the probes were designed against (strain R116265 rather than strain MR766, which is the strain whose reference genome was used for probe design; Fig. 3a and b) and a different species of Human Adenovirus (HAdV) than the probes were designed to target (HAdV-51, a member of species $\mathrm{D}$; as opposed to species $\mathrm{C}, \mathrm{B}$, and $\mathrm{E}$, which the probes target specifically; Fig. $3 g$ and $h$ ). These samples were also constructed to include biological replicates and were probed singly or in pools of four or 12. In all cases, the spiked-in virus was detectable, although there was some variation in depth of coverage among multiplexed samples. As might be expected, samples that were multiplexed in sets of 12 yielded the lowest depth of coverage compared to samples that were multiplexed in sets of four or probed singly (Fig. 3b, d, f). The target genomes were completely covered in the majority of on-target samples. The exception to this rich, consistent coverage included both strains of ZIKV, which, although both were detectable, did not achieve 100\% linear coverage and exhibited lower depth of coverage than the other viruses that were spiked in at similar levels (Fig. 3b).

It should be noted that in this experiment, both purified RNA as well as total nucleic acid samples containing HAdV-4 and HAdV-51 genetic material were processed and sequenced (Fig. $3 \mathrm{~g}$ and $\mathrm{h}$ ). The total nucleic acid samples were processed without a DNase step to allow for potential detection of both the DNA viral genome as 


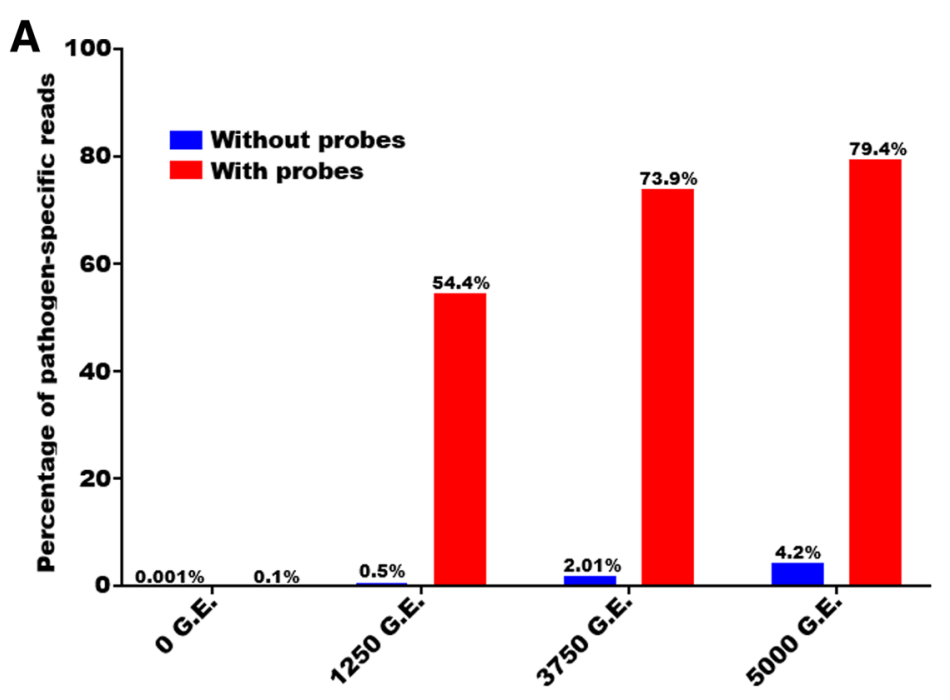

Estimated Influenza A virus genome equivalents per $\mathbf{m L}$ (spiked-in)

\section{B Influenza A virus detected without probes \\ Influenza A virus detected by probes \\ MERS-CoV detected without probes \\ MERS-CoV detected by probes}

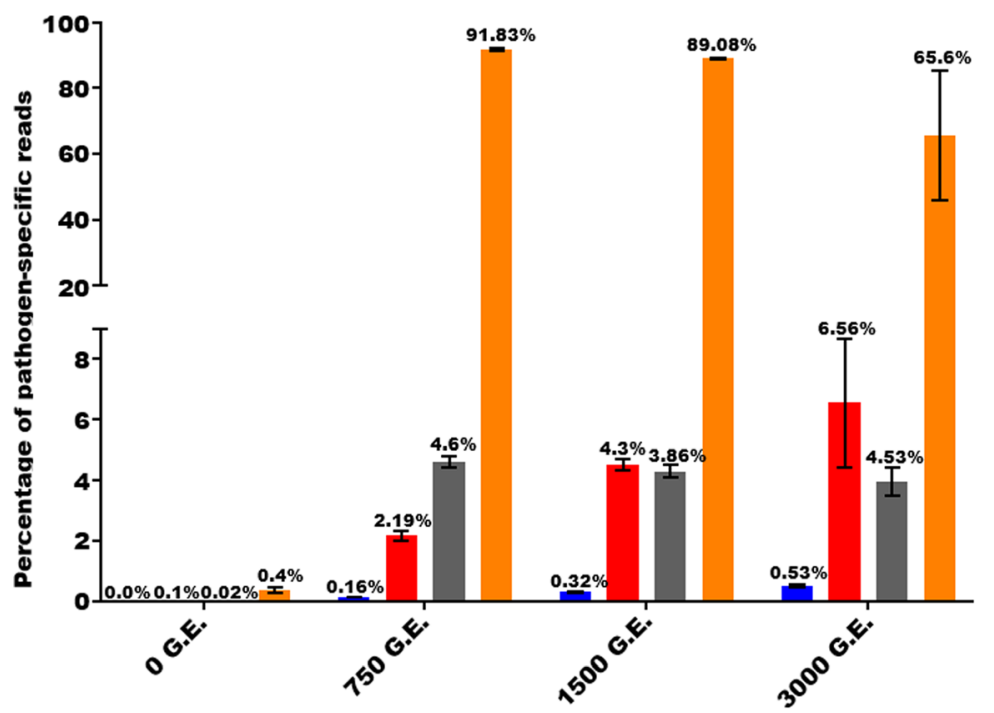

Estimated Influenza A virus genome equivalents per $\mathbf{m L}$ (spiked-in)

Fig. 1 Hybridization-based enrichment enhances sensitivity of HTS for viral detection from complex samples. Known concentrations of IFV genomic RNA (gRNA) were spiked into complex matrices. Samples were split into two parts and processed in parallel via unbiased, shotgun sequencing or target enrichment sequencing in pools of four. a IFV was spiked into bat guano at increasing concentrations to simulate environmental-type samples. Shown here is the percentage of IFV-specific reads. $\mathbf{b}$ Increasing concentrations of IFV gRNA were spiked into total RNA derived from MERS-CoV cell culture lysate. MERS-CoV genomic material was present at a constant, high level amongst all samples. The average percentage of IFV and MERS-CoV virus-specific reads derived from three biological replicates is shown. Black bars denote standard error of the mean for each sample

well as viral transcripts. In the case of both HAdV-4 (species E) and HAdV-51 (species D, not targeted specifically by probes), the vast majority of the resulting sequencing reads were virus-specific and the reads were well-distributed along the length of the genome in coding regions, and in the case of the total nucleic acid samples, noncoding regions as well (Fig. 3h), a phenomenon that was consistent between replicates 


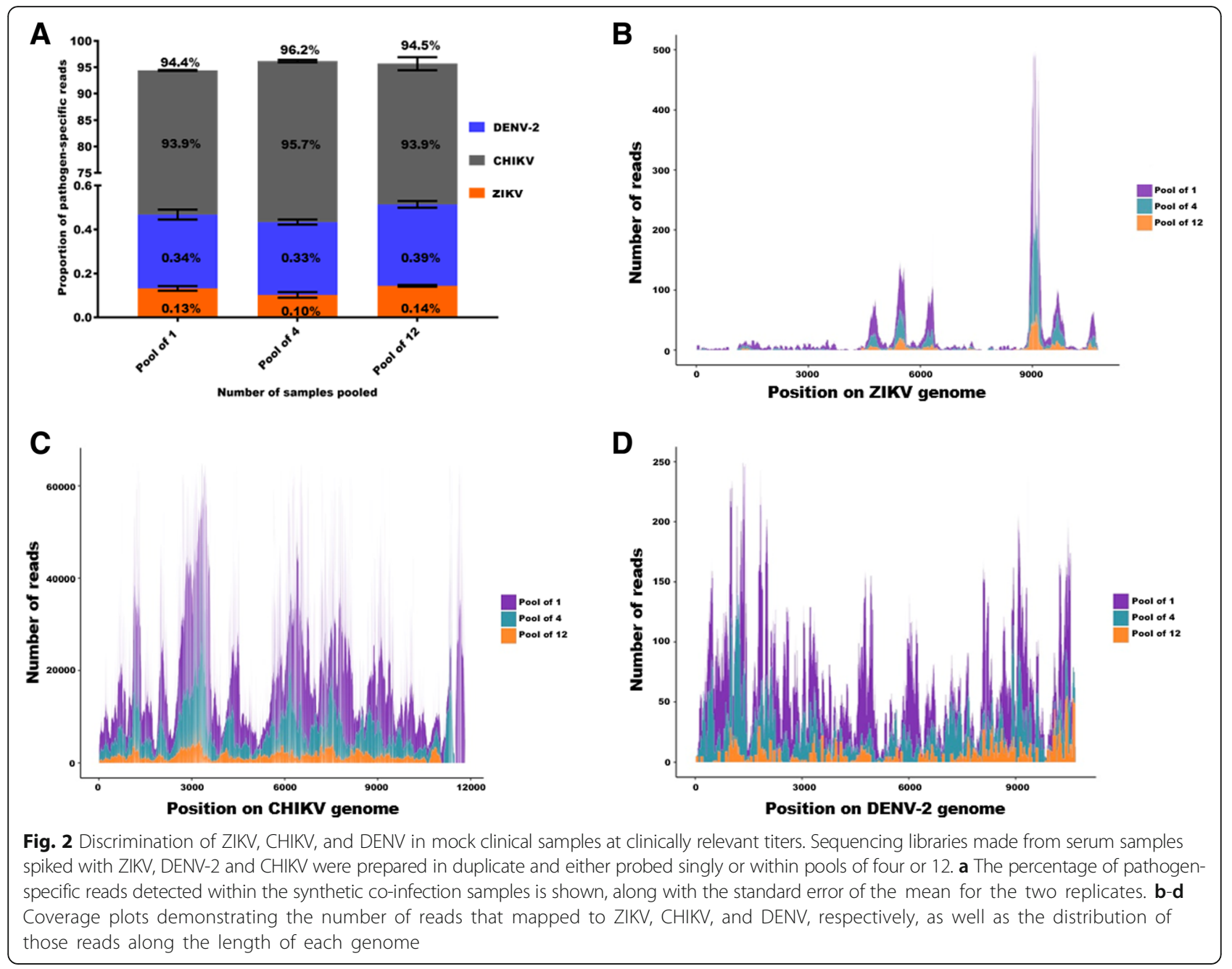

(replicate coverage data is shown in Additional file 4: Figure S2).

\section{Strain-specific detection of DENV at titers below limit of detection by conventional shotgun HTS or amplicon-based sequencing}

It can be difficult to detect DENV-1 and DENV-2 in clinical samples when the Ct value crosses above 29 [5]. Therefore, spiked samples were created using two serotypes of DENV with Ct values corresponding to low titer, and the samples were subjected to hybridization-based enrichment and sequencing. The resulting sequence reads were found to cover the entirety of each target genome, even for the samples corresponding to Ct value 32 (estimated 1000 genome equivalents per $\mathrm{mL}$ ). A dosedependent response was observed in the percentage of DENV-specific reads as the Ct value decreased (Fig. 4a). For each serotype, the depth of coverage was greater than 50x even when the $\mathrm{Ct}$ value crossed 29 (Fig. $4 \mathrm{~b}$ and c). As expected, the remaining reads that did not map to DENV-1 or DENV-2 were derived from human genes in spiked-in human serum RNA extract that were pulled down by the control probes, as well as the sequencing control library for PhiX.

\section{Discussion}

A major challenge faced in virus detection as well as virus sequencing is the difficulty to detect divergent strains of viruses typically present at low titers amongst a robust host or environmental background. Small viral genomes present at low concentrations are effectively drowned out by signal from host nucleic acid and from commensal microorganisms. A variety of methods have been employed to increase the viral signal in highthroughput sequence data, including amplicon sequencing, but for viruses like DENV, with its genome of less than $11 \mathrm{~kb}$ in size, even amplicon sequencing is regarded as an inefficient approach for samples with $\mathrm{Ct}$ values of 29 or higher [5]. Such limitations have been of particular concern for U.S. Department of Defense (DoD) laboratories tasked with biosurveillance and biodefense activities in regions with limited material resources and human 

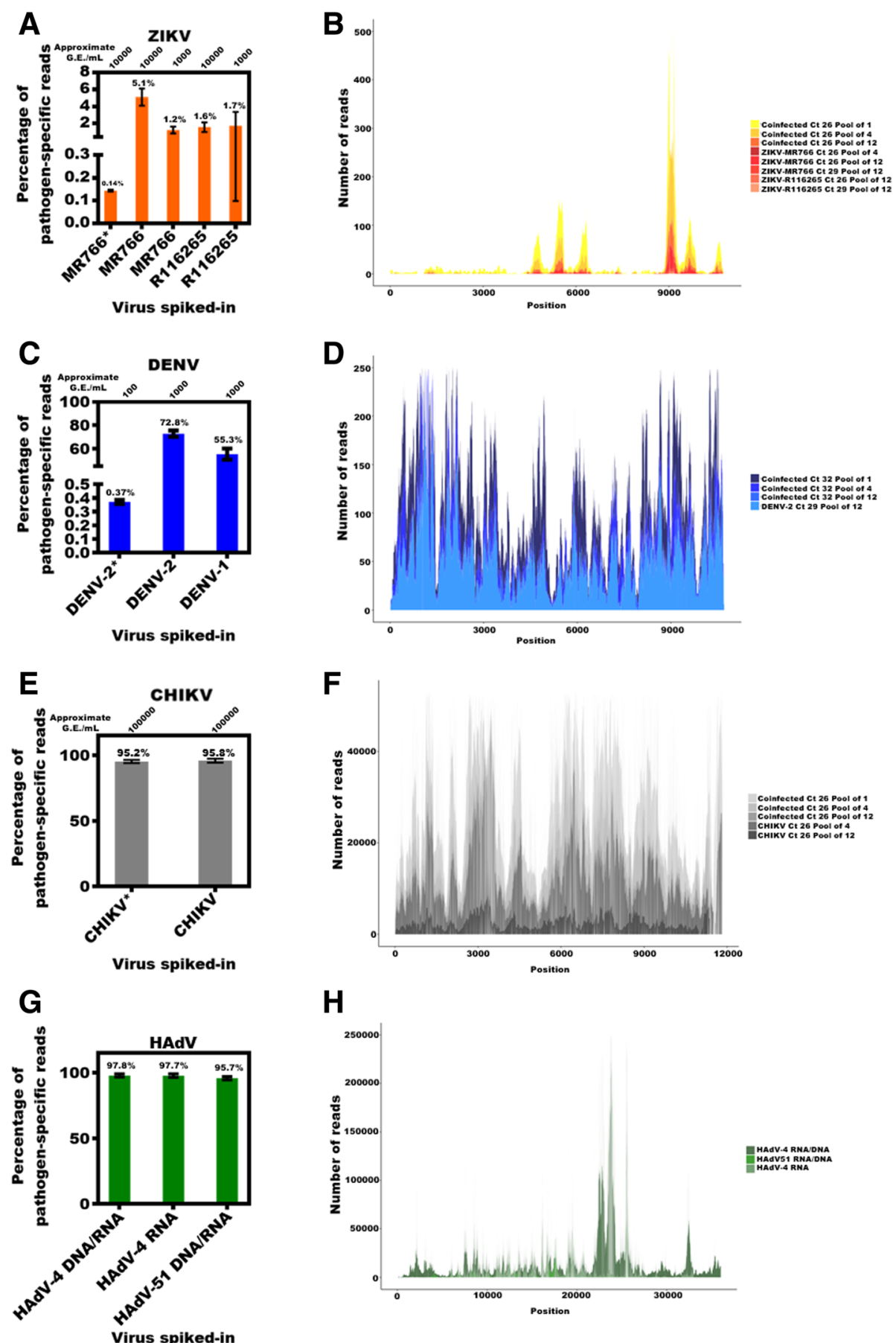

H

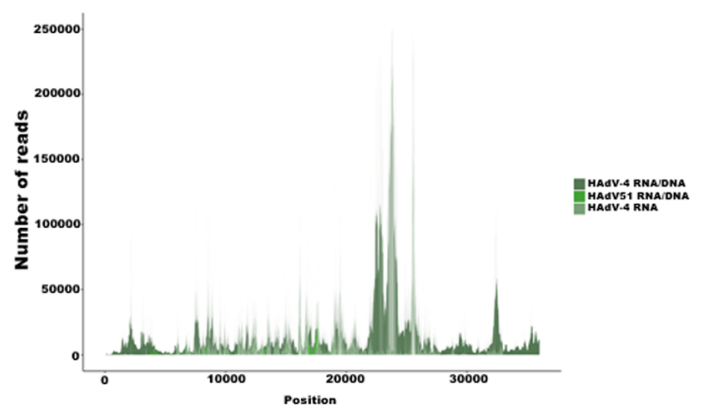

Fig. 3 Detection of close relative viruses irrespective of extensive multiplexing. Sequencing libraries made from serum samples spiked with ZIKV, DENV, CHIKV and/or HAdV were prepared in duplicate and probed singly or probed in pools of four or 12. $\mathbf{a}, \mathbf{c}, \mathbf{e}, \mathbf{g}$ The percentage of reads that map to each strain of spiked-in ZIKV, DENV, CHIKV, and HAdV, respectively. Each co-infected sample is denoted with an asterisk (*). Estimated genome equivalents per $\mathrm{mL}$ as extrapolated from RT-qPCR standard curves are listed along the top of each graph. The standard error of the mean of two replicates is shown. $\mathbf{b}, \mathbf{d}, \mathbf{f}, \mathbf{h}$ Coverage plot for replicate one of each ZIKV-, DENV-2-, CHIKV-, and HAdV-containing sample, respectively

expertise. Part of the motivation for this effort was to provide DoD laboratories operating in austere environments new tools aimed at enhancing on-site sequencing capacity when engaged in Force Health Protection (FHP) activities.
We demonstrate here that hybridization-based viral target enrichment yields robust coverage of small genomes from clinical samples, even yielding full-length, deeply covered genomes at concentrations whereby 


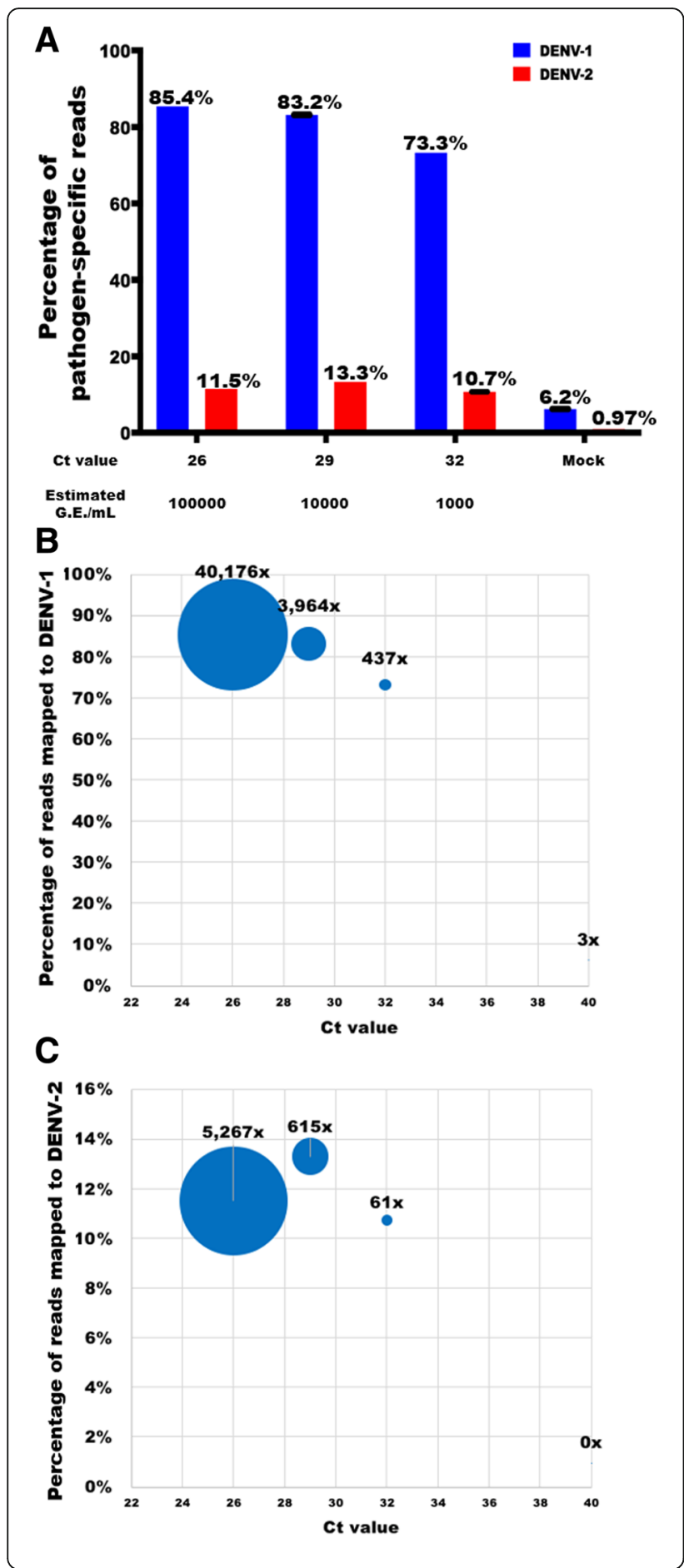

Fig. 4 Recovery of full DENV genome at titers below limit of detection by conventional shotgun HTS or amplicon-based sequencing. DENV-1 and DENV-2 RNA were spiked into human serum RNA at a range of GE corresponding to Ct 26-32, in duplicate, and libraries were prepared using target enrichment in pools of four. Corresponding estimated genome equivalents per $\mathrm{mL}$ as extrapolated from a standard RT-qPCR curve are listed below the axis. Mock samples consisted of human serum RNA extract only. a The proportion of total reads that map to DENV-1 or DENV-2 at each Ct value. Error bars show standard error of two replicates. $\mathbf{b}-\mathbf{c}$ The proportion of reads that map to DENV-1 or DENV-2, respectively, at each spike-in level. Bubble size corresponds to depth of coverage of the viral genome (average of two replicates)

current amplicon sequencing protocols may be expected to fail. Moreover, we demonstrate that hybridizationbased target enrichment can allow for not only detection, but also genetic characterization such as strainlevel discrimination, even at very low concentrations of virus. The capability to detect and discriminate between multiple serotypes of a virus within a complex sample at clinically relevant concentrations by using this enrichment method increases the utility of high throughput sequencing for biosurveillance and for infectious disease diagnostics. For both biosurveillance and clinical sequencing, assay cost and time are important considerations. We have demonstrated that more extensive pooling and multiplexing can be performed to reduce cost and time without sacrificing the assay's ability to detect at least two strains of related virus and a variety of unrelated viruses in one sequencing reaction.

To date, the published viral target enrichment studies vary in focus and include characterization of EBOV during a recent outbreak in West Africa $[9,10]$ and detection of multiple viral families within clinical samples $[13,16]$. While the probes employed in the studies published to date vary in length from 50- to 120-mers, enrichment methods also can differ by the number of probes and target viruses included in a set. An additional potential protocol difference is the number of samples pooled, which ranges from a single sample to $12[9,10,15]$. The current recommendation by Illumina for viral enrichment is to pool four samples [14].

The experiments described here systematically test enrichment of a single library as well as pools of four or 12 libraries and include a variety of titers of as many as three viruses within a single sample and as many as 12 samples within an enriched pool. For all conditions, even with more extensive pooling and multiplexing, we observed a dose-dependent response to varying $\mathrm{Ct}$ values even in co-infected clinical samples. A dose dependent response was also observed by O'Flaherty et al. in two co-infected samples containing Respiratory Syncytial virus and human Coronavirus OC43 spiked-in each at Ct 28 or 32 [13]. Interestingly, although there was the expected dose-dependent effect on the proportion of 
sequencing reads derived from IFV as the concentration of spiked IFV gRNA increased, there was a slight decrease in the proportion of MERS-CoV-derived sequencing reads in samples at the upper end of the IFV gRNA concentration range. This was not expected given that MERS-CoV was present in each replicate at a constant, high level. We hypothesize that this may be due to saturation of the streptavidin beads used to capture probe-cDNA hybridized fragments. Further experimentation will be required to test that hypothesis.

Efficacy of probes varies by homology to the viral target, as evidenced by our results and those in the literature $[13,15]$. For example, the reference sequence used to design the probe set for DENV-1 exhibits $74 \% \mathrm{nu}-$ cleotide identity over $35 \%$ of the length of the closest sequenced reference for the DENV-2 strain that was spiked. It is possible that this overlap, which is not shared by the DENV-2 probes and DENV-1 spike-in, contributed to an overrepresentation of DENV-1 reads in the experiments presented in Figs. 3 and 4. Additionally, by comparison to the other richly covered strains of virus tested in multiplexed samples, there was an underwhelming coverage for both strains of ZIKV. It is possible that the quality of RNA from these viruses was less than the other RNA spike-ins, or that the probe panel for ZIKV is less efficacious when used in combination with the entirety of the probe set. The probes for ZIKV were synthesized and added later after all the other probes were combined (in response to the recent outbreak) and therefore it is possible that the comparatively lower performance of the ZIKV probes is due to a difference in quantitation of the ZIKV probe set.

We observed that HAdV-51 (species D) genetic material was efficiently enriched even though the only adenoviruses used to design the probe panel were species $B, C$, and $\mathrm{E}$. This experiment indicates that the protocol works as well for this particular DNA virus as it does for RNA viruses. Limits of detection may vary by viral target, which may explain why previously published experiments showed differences between DNA and RNA viruses [13]. Nucleotide identity between human adenovirus species $D$ (the species to which HAdV- 51 belongs) and the other human adenovirus species HAdV-A, B, C, and $\mathrm{E}$ was reported by Kaneko et al. to range from 58.73 to $69.35 \%$ [26]. In our study, the probe panel containing probes for species HAdV-B, C, and E effectively enriched for the entirety of the HAdV-D genome. This suggests that when using long (80-mer) probes designed against several species of virus, related non-targeted species may also be enriched without being specifically included in the panel, if the nucleotide identity among them is at least $60-70 \%$, and if multiple related species are targeted by the probes in the panel (in this case three species). This cross-reactivity for related human pathogens could prove to be a useful feature, by allowing for enrichment of more relevant viruses without added cost spent to increase the number of probes.

Our findings demonstrate that breadth of coverage does not suffer from extensive pooling but that deeper depth of coverage is gained by limiting the number of samples pooled. Extensive pooling makes hybridizationbased enrichment sequencing more economical. Viral target enrichment could be applied as an economical approach to sequencing viruses known to mutate quickly and therefore evade other assays, fastidious organisms, or complex samples of limited volume. For example, this method could be prescribed to a scenario in which multiple serotypes of a virus such as DENV are expected to be present in a sample but detection is prohibited via conventional methods such as amplicon sequencing due to low titers. Viral target enrichment designed for a broad panel of targets could also be useful to the infectious disease field by enabling detection of low-titer viruses present in clinical samples taken from patients suffering from symptoms of unknown etiology. Another applicable use of a broad probe panel could be to perform environmental sampling. The aforementioned applications often involve complex samples of limited volume, for which this method is ideal. An important caveat to this approach is that while viral target enrichment is an economical method by which to reduce background noise in a metagenomic sample, probe design requires prior knowledge of the closest-sequenced genome for each viral target. Amplicon sequencing may be the best approach for previously known samples and unbiased whole shotgun sequencing may be more appropriate for a virus-rich sample. None of these approaches obviates the use of amplification by polymerase chain reaction or the potential introduction of sequence errors, and so standard quality analyses by computational methods should always be employed. As the development and testing of probe sets for viral target enrichment expands and continues, the application of this technique could make HTS more economical for routine use in Force Health Protection activities including biosurveillance, biodefense and outbreak investigations.

\section{Methods}

Preparation of contrived metagenomic samples and nucleic acid extraction and quality control IFV (H1N1) particles (A/Swine/Iowa/15/30; ATCC, Manassas, VA), MERS-CoV RNA (Jordan-N3/2012; NAMRU-3), HAdV nucleic acid extract from particles (RI-67 and Bom; ATCC, Manassas, VA), ZIKV RNA extract from particles (MR766 and R116265; ATCC, Manassas, VA), CHIKV RNA (gift from LTC Richard Jarman, Walter Reed Army Institute of Research), DENV-1 RNA extract from particles (TH-SMAN; ATCC, Manassas, VA) and DENV-2 RNA 
extract from particles (New Guinea C; ATCC, Manassas, VA) were spiked into relevant matrices to construct contrived metagenomic samples for testing.

To prepare guano samples five-gram quantities of commercial Jamaican bat guano (Planet Natural; Bozeman, MT) were placed in $50 \mathrm{~mL}$ of sterile-filtered Hank's Balanced Salt Solution (HBSS), vortexed to mix, centrifuged at 3,100 x g for $10 \mathrm{~min}$, and filtered sequentially through $0.45 \mu \mathrm{m}$ and $0.22 \mu \mathrm{m}$ filters prior to spiking with IFV particles. Post-addition of IFV, samples were centrifuged at $39,000 \times \mathrm{g}$ for three hours at $10^{\circ} \mathrm{C}$ to concentrate spiked and native virus particles, supernatant was removed, and total RNA was extracted from the pellet using the QIAampViral RNA Isolation Kit (QIAGEN; Valencia, CA). After elution in $30 \mu \mathrm{L}$ buffer AVE, a second elution using $20 \mu \mathrm{L}$ of the eluate was performed.

To prepare cell culture matrix samples, a nucleic acid spiking approach was used. In this case, decreasing amounts of IFV RNA were spiked into a constant mass of total RNA that had been extracted from Vero cells infected with MERS-CoV. Aliquots of the same Vero cell culture were used for each sample. Genome equivalents of IFV spiked into samples were calculated based on RNA mass extracted from virus particles and genome size.

For serum samples, RNA was extracted from Human Serum (BioIVT, Westbury, NY) and mixed with viral RNA.

Total nucleic acid was extracted from adenovirus particles using the QIAGEN QiAMP MinElute Virus Spin Kit, omitting carrier RNA. The samples were eluted in $24 \mu \mathrm{l}$ buffer AVE. Viral RNA was extracted from virus particles and human serum using the QIAampViral RNA Isolation Kit as described above. The Qubit double stranded DNA Broad-Range Assay Kit and the Qubit RNA Broad-Range Assay Kit (Thermo Fisher Scientific; Waltham, MA) were used to assay extracts.

\section{Quantitative reverse transcription PCR}

For quantitative reverse transcription PCR, SuperScriptIII RT/Platinum Taq Mix (Thermo Fisher Scientific; Waltham, MA), dNTP mix, $\mathrm{MgSO}_{4}$, ROX Reference Dye
(Thermo Fisher Scientific; Waltham, MA), and the primers and probes listed in Table 2 were used to assay in the CFX Connect Real-Time PCR Detection System (Bio-Rad; Hercules, CA) using the following conditions: $50^{\circ} \mathrm{C}$ for $15 \mathrm{~min}, 95^{\circ} \mathrm{C}$ for two minutes, and 40 cycles of $95^{\circ} \mathrm{C}$ for $15 \mathrm{~s}$ and $60^{\circ} \mathrm{C}$ for one minute. Standard curves for DENV-1, DENV-2, CHIKV and ZIKV were generated from titrated viral RNA extract.

\section{Library preparation, virus enrichment, and sequencing}

For virus enriched sequencing, TruSeq RNA Access libraries were created as per manufacturer's protocol (Illumina; San Diego, CA), with the following two modifications: i) rather than the standard CEX oligonucleotides that are designed for enrichment of human genes, a custom pool of oligonucleotides was used that includes probes along the entire genome length of 83 viruses (Table 1) as well as probes specific for several human house-keeping genes, and ii) in the second PCR amplification, 17 cycles were used rather than ten. Samples were probed singly or in pools of four or 12 and multiplexed for sequencing on the Illumina MiSeq platform using v3 chemistry, 2X75 bp read lengths.

For conventional HTS (shotgun) sequencing, TruSeq libraries were pooled and sequenced on the MiSeq platform using v3 chemistry, 2X300 bp read lengths. For the guano samples, these consisted of an aliquot of each of the TruSeq RNA Access libraries from the step prior to virus enrichment. For the MERS-CoV-Vero cell matrix samples, these consisted of shotgun libraries made with the TruSeq RiboZero Gold Library Preparation Kit (Illumina; San Diego, CA).

\section{Virus enrichment probe design}

A composite panel of 80-mer DNA probes was assembled using a previously described panel for respiratory viruses [13, 14, 20], a previously described panel for Filoviruses [9-11], plus an additional panel of newly designed probes for 41 viruses of biosurveillance and biodefense concern, for a total of 19,077 probes. The methods employed for capture oligo design were

Table 2 Primers and probes used for qRT-PCR

\begin{tabular}{|c|c|c|c|c|}
\hline Virus & Forward Primer Sequence & Reverse Primer Sequence & Probe Sequence & Reference \\
\hline CHIKV (181/Clone 25) & AGCTCCGCGTCCTTTACCA & $\begin{array}{l}\text { GCCAAATTGTCCTGGT } \\
\text { CTTCCT }\end{array}$ & Express One SYBR Green I (Life Technologies) & {$[30]$} \\
\hline DENV-1 (TH-SMAN) & GACACCACACCCTITGGACAA & $\begin{array}{l}\text { CACCTGGCTGTCAC } \\
\text { CTCCAT }\end{array}$ & $\begin{array}{l}\text { FAM-AGAGGGTGTTTAAAGAGAAAGTTGACACG } \\
\text { CG-TAMRA }\end{array}$ & [31] \\
\hline DENV-2 (New Guinea C) & ACAGGCTATGGCACTGTTACGAT & $\begin{array}{l}\text { TGCAGCAACACCAT } \\
\text { CTCATTG }\end{array}$ & FAM-AGTGCTCTCCAAGAACGGGCCTCG-TAMRA & {$[32,33]$} \\
\hline $\begin{array}{l}\text { IFV-A - HA (A/Swine/ } \\
\text { lowa/15/30) }\end{array}$ & CCAGTCACAATAGGAGAGTG & $\begin{array}{l}\text { AAACCGGCAATGGC } \\
\text { TCCAAA }\end{array}$ & Express One SYBR Green I (Life Technologies) & [34] \\
\hline ZIKV (MR766 and R116265) & $\begin{array}{l}\text { AARTACACATACCARAACA } \\
\text { AAGTGGT }^{\text {a }}\end{array}$ & $\begin{array}{l}\text { TCCRCTCCCYCTYTGGT } \\
\text { CTTG }\end{array}$ & Express One SYBR Green I (Life Technologies) & [35] \\
\hline
\end{tabular}

aboldface $T$ was modified from originally published $R$ 
essentially as described in O'Flaherty at al [13], although the design varied somewhat across the target viral genomes. For instance, in the case of the previously described respiratory virus panel, the design was focused on coding regions [13]. In general, genomes were tiled with capture oligos in a way so as to avoid low-complexity sequences and repetitive sequences. Probe spacing and overlap vary per virus due to attempts to design probes that cover multiple related virus strains resulting in overlapping tiled design around more variable regions, whereas regions more conserved among multiple strains resulted in probes more or less tiled end-to-end. All probes were biotinylated on the $5^{\prime}$ end. Sequences of viral capture probes are provided in Additional file 5.

\section{Bioinformatic analyses}

Quality control, de novo assembly, taxonomic classification, and reference-based analyses were conducted using EDGE Bioinformatic software v 2.0 [27] with default parameters and host removal of human reference GRCh38 and also with CLC Genomics Workbench v11 (QIAGEN Bioinformatics; Redwood City, CA). The reference mapping parameters in CLC were modified from defalt settings to 0.8 length fraction and 0.8 similarity fraction with global alignment and random mapping of non-specific matches. BLAST [28] was also used to further investigate specific datasets. The ggplot2 $R$ package was used to generate depth of coverage plots [29].

\section{Additional files}

Additional file 1: Table S1. Number and proportion of reads mapped to IFV at spiked-in genome equivalents of $0,1,250,3,750$ and 5,000 given preparation by hybridization-based target enrichment or shotgun sequencing. (DOCX $12 \mathrm{~kb}$ )

Additional file 2: Table S2. Number and proportion of reads mapped to IFV or MERS-CoV at IFV spiked-in genome equivalents of 0, 750, 1,500 and 3,000 and a constant, high level of MERS-CoV genomic material given preparation by hybridization-based target enrichment or shotgun sequencing. (DOCX $15 \mathrm{~kb}$ )

Additional file 3: Figure S1. Coverage plots demonstrating the number of reads that mapped to ZIKV, CHIKV, and DENV, respectively, as well as the distributionof those reads along the length of each genome. Replicates shown in Figure 2. (TIF $338 \mathrm{~kb}$ )

Additional file 4: Figure S2. Coverage plot for replicate one of each ZIKV-, DENV-2-, CHIKV-, and HAdV-containing sample, respectively. Replicates shown in Figure 3. (TIF $655 \mathrm{~kb}$ )

Additional file 5: Nucleotide sequences for the custom pool of oligonucleotides that includes probes along the entire genome length of 83 viruses. (XLSX $1043 \mathrm{~kb})$

\section{Abbreviations}

bp: base-pair; CEX: Coding exome; CFU: Colony-forming units; CHIKV: Chikungunya virus; Ct: Cycle threshold; DENV: Dengue virus; DNA: Deoxyribonucleic acid; ds: Double-stranded; EBOV: Ebola virus; FFPE: Formalin-fixed paraffin-embedded; GE: Genome equivalents; gRNA: Genomic RNA; HA: Hemagglutinin; HAdV: Human Adenovirus; HTS: High-Throughput Sequencing; IFV: Influenza virus; kb: kilobase; MERS-CoV: Middle Eastern Respiratory Syndrome Coronavirus; NGS: Next-
Generation Sequencing; PCR: Polymerase Chain Reaction; PFU: Plaque-forming units; RNA: Ribonucleic Acid; RT-PCR: Real-time PCR; RT-qPCR: Reverse transcription quantitative PCR; RVP: Respiratory Virus Panel; ss: Single-stranded; ZIKV: Zika virus

\section{Acknowledgements}

The authors would like to thank LCDR Gabriel Defang from NMRC for providing samples of MERS-CoV Vero cell culture matrix and LTC Richard Jarman of WRAIR for providing CHIKV RNA.

\section{Funding}

This work was funded by U.S. Navy, Office of Naval Research, In-House Laboratory Independent Research (ILIR) Program and WUN A1417. The funding bodies had no role in the design of this study nor in the collection, analysis, or interpretation of data, nor in the writing of this manuscript.

\section{Availability of data and materials}

The datasets supporting the conclusions of this article are available in National Center for Biotechnology Information (NCBI) Sequence Read Archive (SRA) as PRJNA494391.

\section{Authors' contributions}

$A C P, K G F, G S, S G$, and TH performed the experiments. ACP, KGF, and KAB-L conceived the experimental design and analyzed the data. ACP and KAB-L wrote the manuscript. All authors read and approved the final manuscript.

\section{Authors' information}

The views expressed in this manuscript are those of the authors and do not necessarily reflect the official policy or position of the Department of the Navy, the Department of Defense, nor the U.S. Government. TH is a military service member; KGF, and KAB-L are employees of the U.S. Government. This work was prepared as part of their official duties. Title 17 U.S.C. §105 provides that 'Copyright protection under this title is not available for any work of the United States Government.' Title 17 U.S.C. §101 defines a U.S. Government work as a work prepared by a military service member or employee of the U.S. Government as part of that person's official duties.

Ethics approval and consent to participate

Not applicable

\section{Consent for publication}

Not applicable

\section{Competing interests}

The authors declare that they have no competing interests.

\section{Publisher's Note}

Springer Nature remains neutral with regard to jurisdictional claims in published maps and institutional affiliations.

\section{Author details}

${ }^{1}$ Genomics and Bioinformatics Department, Biological Defense Research Directorate, Naval Medical Research Center - Frederick, Fort Detrick, Frederick, MD 21702, USA. ${ }^{2}$ Uniformed Services University of the Health Sciences, Bethesda, MD 20814, USA. ${ }^{3}$ Leidos, Reston, VA 20190, USA. ${ }^{4}$ Illumina Inc., San Diego, CA 92122, USA.

Received: 30 October 2018 Accepted: 18 February 2019

Published online: 26 February 2019

\section{References}

1. Frey KG, Bishop-Lilly KA: Next-generation sequencing for pathogen detection and identification. In: Methods Microbiol vol. 42, 31 July 2015 edn: Elsevier Ltd: 2015: 525-554.

2. Thurber RV, Haynes M, Breitbart M, Wegley L, Rohwer F. Laboratory procedures to generate viral metagenomes. Nat Protoc. 2009;4(4):470-83.

3. Metsky HC, Matranga CB, Wohl S, Schaffner SF, Freije CA, Winnicki SM, West K, Qu J, Baniecki ML, Gladden-Young A, et al. Zika virus evolution and spread in the Americas. Nature. 2017.

4. Quick J, Grubaugh ND, Pullan ST, Claro IM, Smith AD, Gangavarapu K, Oliveira G, Robles-Sikisaka R, Rogers TF, Beutler NA, et al. Multiplex PCR 
method for MinION and Illumina sequencing of Zika and other virus genomes directly from clinical samples. Nat Protoc. 2017;12(6):1261-76.

5. Cruz CD, Torre A, Troncos G, Lambrechts L, Leguia M. Targeted full-genome amplification and sequencing of dengue virus types 1-4 from South America. J Virol Methods. 2016;235:158-67.

6. Leguia M, Cruz CD, Felices V, Torre A, Troncos G, Espejo V, Guevara C, Mores C. Full-genome amplification and sequencing of Zika viruses using a targeted amplification approach. J Virol Methods. 2017;248:77-82.

7. Samorodnitsky E, Jewell BM, Hagopian R, Miya J, Wing MR, Lyon E, Damodaran S, Bhatt D, Reeser JW, Datta J, et al. Evaluation of hybridization capture versus amplicon-based methods for whole-exome sequencing. Hum Mutat. 2015;36(9):903-14.

8. Sozhamannan S, Holland MY, Hall AT, Negron DA, Ivancich M, Koehler JW, Minogue TD, Campbell CE, Berger WJ, Christopher GW, et al. Evaluation of signature Erosion in Ebola virus due to genomic drift and its impact on the performance of diagnostic assays. Viruses. 2015;7(6):3130-54.

9. Blackley DJ, Wiley MR, Ladner JT, Fallah M, Lo T, Gilbert ML, Gregory C, D'Ambrozio J, Coulter S, Mate $\mathrm{S}$, et al. Reduced evolutionary rate in reemerged Ebola virus transmission chains. Sci Adv. 2016;2(4):e1600378.

10. Mate SE, Kugelman JR, Nyenswah TG, Ladner JT, Wiley MR, Cordier-Lassalle T, Christie A, Schroth GP, Gross SM, Davies-Wayne GJ, et al. Molecular evidence of sexual transmission of Ebola virus. N Engl J Med. 2015;373(25): 2448-54.

11. Luke T, Bennett RS, Gerhardt DM, Burdette T, Postnikova E, Mazur S, Honko AN, Oberlander N, Byrum R, Ragland D, et al. Fully human immunoglobulin $\mathrm{G}$ from Transchromosomic bovines treats nonhuman Primates infected with Ebola virus Makona isolate. J Infect Dis. 2018.

12. Illumina: TruSeq RNA access library prep guide. In: Cold Spring Harbor Protocols. 31 march 2014 edn; 2014.

13. O'Flaherty BM, Li Y, Tao Y, Paden CR, Queen K, Zhang J, Dinwiddie DL Gross SM, Schroth GP, Tong S. Comprehensive viral enrichment enables sensitive respiratory virus genomic identification and analysis by next generation sequencing. Genome Res. 2018;28(6):869-77.

14. Yang $Y$, Walls SD, Gross SM, Schroth GP, Jarman RG, Hang J. Targeted sequencing of respiratory viruses in clinical specimens for pathogen identification and genome-wide analysis. Methods Mol Biol. 2018;1838: 125-40.

15. Metsky HC, Siddle KJ, Gladden-Young A, Qu J, Yang DK, Brehio P, Goldfarb A, Piantadosi A, Wohl S, Lin AE, et al. Capturing diverse microbial sequence with comprehensive and scalable probe design. In: bioRxiv (preprint); 2018

16. Cummings MJ, Tokarz R, Bakamutumaho B, Kayiwa J, Byaruhanga T, Owor N, Namagambo B, Wolf A, Mathema B, Lutwama JJ, et al. Precision surveillance for viral respiratory pathogens: virome capture sequencing for the detection and genomic characterization of severe acute respiratory infection in Uganda. Clin Infect Dis. 2018.

17. Wylie TN, Wylie KM, Herter BN, Storch GA. Enhanced virome sequencing using targeted sequence capture. Genome Res. 2015;25(12):1910-20.

18. Oba M, Tsuchiaka S, Omatsu T, Katayama Y, Otomaru K, Hirata T, Aoki H, Murata $Y$, Makino S, Nagai M, et al. A new comprehensive method for detection of livestock-related pathogenic viruses using a target enrichment system. Biochem Biophys Res Commun. 2018;495(2):1871-7.

19. Brown JR, Roy S, Ruis C, Yara Romero E, Shah D, Williams R, Breuer J. Norovirus whole-genome sequencing by SureSelect target enrichment: a robust and sensitive method. J Clin Microbiol. 2016;54(10):2530-7.

20. Dehority WN, Eickman MM, Schwalm KC, Gross SM, Schroth GP, Young SA Dinwiddie DL. Complete genome sequence of a KI polyomavirus isolated from an otherwise healthy child with severe lower respiratory tract infection. J Med Virol. 2017:89(5):926-30

21. Paixao ES, Teixeira MG, Rodrigues LC. Zika, chikungunya and dengue: the causes and threats of new and re-emerging arboviral diseases. BMJ Glob Health. 2018;3(Suppl 1):e000530.

22. Wang WK, Sung TL, Tsai YC, Kao CL, Chang SM, King CC. Detection of dengue virus replication in peripheral blood mononuclear cells from dengue virus type 2-infected patients by a reverse transcription-realtime PCR assay. J Clin Microbiol. 2002;40(12):4472-8.

23. Corman VM, Rasche A, Baronti C, Aldabbagh S, Cadar D, Reusken CB, Pas SD, Goorhuis A, Schinkel J, Molenkamp R, et al. Assay optimization for molecular detection of Zika virus. Bull World Health Organ. 2016; 94(12):880-92.
24. Panning M, Grywna K, van Esbroeck M, Emmerich P, Drosten C. Chikungunya fever in travelers returning to Europe from the Indian Ocean region, 2006. Emerg Infect Dis. 2008;14(3):416-22.

25. Lanciotti RS, Kosoy OL, Laven JJ, Velez JO, Lambert AJ, Johnson AJ, Stanfield SM, Duffy MR. Genetic and serologic properties of Zika virus associated with an epidemic, yap state, Micronesia, 2007. Emerg Infect Dis. 2008;14(8):1232-9.

26. Kaneko H, lida T, Ishiko H, Ohguchi T, Ariga T, Tagawa Y, Aoki K, Ohno S, Suzutani T. Analysis of the complete genome sequence of epidemic keratoconjunctivitis-related human adenovirus type 8, 19, 37 and a novel serotype. J Gen Virol. 2009;90(Pt 6):1471-6.

27. Li PE, Lo CC, Anderson JJ, Davenport KW, Bishop-Lilly KA, Xu Y, Ahmed S, Feng S, Mokashi VP, Chain PS. Enabling the democratization of the genomics revolution with a fully integrated web-based bioinformatics platform. Nucleic Acids Res. 2017:45(1):67-80.

28. Altschul SF, Gish W, Miller W, Myers EW, Lipman DJ. Basic local alignment search tool. J Mol Biol. 1990;215(3):403-10.

29. Wickham H. ggplot2: elegant graphics for data analysis. New York: SpringerVerlag; 2016.

30. van Duijl-Richter MKS, Blijleven JS, van Oijen AM, Smit JM. Chikungunya virus fusion properties elucidated by single-particle and bulk approaches. J Gen Virol. 2015;96(8):2122-32.

31. Callahan JD, Wu SJ, Dion-Schultz A, Mangold BE, Peruski LF, Watts DM, Porter KR, Murphy GR, Suharyono W, King CC, et al. Development and evaluation of serotype- and group-specific fluorogenic reverse transcriptase PCR (TaqMan) assays for dengue virus. J Clin Microbiol. 2001;39(11):4119-24.

32. Ruiz Silva M, Aguilar Briseno JA, Upasani $V$, van der Ende-Metselaar $H$, Smit JM, Rodenhuis-Zybert IA. Suppression of chikungunya virus replication and differential innate responses of human peripheral blood mononuclear cells during co-infection with dengue virus. PLoS Negl Trop Dis. 2017;11(6): e0005712.

33. van der Schaar HM, Rust MJ, Waarts BL, van der Ende-Metselaar H, Kuhn RJ, Wilschut J, Zhuang X, Smit JM. Characterization of the early events in dengue virus cell entry by biochemical assays and single-virus tracking. J Virol. 2007;81(21):12019-28.

34. Frey KG, Herrera-Galeano JE, Redden CL, Luu TV, Servetas SL, Mateczun AJ, Mokashi VP, Bishop-Lilly KA. Comparison of three next-generation sequencing platforms for metagenomic sequencing and identification of pathogens in blood. BMC Genomics. 2014;15:96.

35. Faye O, Faye O, Diallo D, Diallo M, Weidmann M, Sall AA. Quantitative realtime PCR detection of Zika virus and evaluation with field-caught mosquitoes. Virol J. 2013:10:311.

Ready to submit your research? Choose BMC and benefit from:

- fast, convenient online submission

- thorough peer review by experienced researchers in your field

- rapid publication on acceptance

- support for research data, including large and complex data types

- gold Open Access which fosters wider collaboration and increased citations

- maximum visibility for your research: over $100 \mathrm{M}$ website views per year

At $\mathrm{BMC}$, research is always in progress.

Learn more biomedcentral.com/submission 